\title{
Persistence of pyrethroid Deltamethrin and its effects on extra virgin olive oil quality
}

\author{
Ikram Jaabiri Kamoun $^{1^{*}}$, Dalel Belhaj ${ }^{2}$, Nesrine Turki ${ }^{3}$, Monem Kallel ${ }^{4}$, \\ Habib Ayadi ${ }^{5}$, Mohieddine Ksantini ${ }^{6}$, Jalel Bouzid ${ }^{7}$, Radhia Gargouri ${ }^{8}$ \\ ${ }^{1-2-3-4-7}$ University of Sfax-Tunisia, ENIS. Laboratory of Water- Energy-Environment. Street Soukra Km 3.5. BP \\ 1173 CP 3038. \\ ${ }^{5}$ University of Sfax-Tunisia, Faculty of Sciences, Department of Life Sciences, Laboratory of Biodiversity and \\ Aquatic Ecosystems, Ecology and Planktonology, Street Soukra Km 3.5 BP 1171 CP 3000. \\ ${ }^{6}$ University of Sfax-Tunisia, Laboratory of Grown Plant Protection and Environment, Institute of Olivier, BP \\ 1087 CP 3000. \\ ${ }^{8}$ University of Sfax-Tunisia, ENIS. Laboratory of Plant Biotechnologies applied to crop improvement Street \\ Soukra Km 3.5. BP 1173 CP 3038.
}

\begin{abstract}
The use of pesticides in developing countries remains out of control in major cases. Consequently, it is common to find amounts of residues exceeding the Maximum Residue Limits (MRLs). This work intends to assess the persistence of Deltamethrin and its impact on the olive oil quality. Time of sampling and concentration of treatment were varied. Analyses were performed weekly during two months. The results show that Deltamethrin levels in oil of treated plants declined by about 50\% after 15 days from insecticide application date (1.6 $\left.\mathrm{mg} \mathrm{kg}^{-1}\right)$ for samples treated with the recommended dose or below. No significant differences in oil physicochemical and nutritional parameters were observed. The sensory evaluation of oils extracted after 2 months did not show significant differences in unpleasant aromatic notes nor defects. Olive oil obtained from samples- where the recommended dose was exceeded - had a slower degradation of the active substance. Moreover, some physicochemical parameters of the latters were influenced and the sensory evaluation revealed some defects.

Our results confirm that serious doubts remain about the safety and healthiness of oils extracted from drupes treated with Deltamethrin in developing countries in general, and in Tunisia in particular, where recommended doses are not usually respected. This information could evaluate the real risk in the use of this product for plant protection in olive growing.
\end{abstract}

Keywords: Olive oil quality, Residue, Persistence, Pesticide, pyrethroid

\section{Introduction}

The olive fly (Bactrocera oleae) is one of the principal threats of the quality of olive fruit as it alters its chemical composition [1]. Therefore, among the eligible products for the protection against olive pathogens, Deltamethrin has been widely used in controlling insect pests of medical and agricultural importance [2].

Deltamethrin is an alpha-cyano type-II synthetic pyrethroid; that has become a major class of active insecticides due to high insecticidal potency. The exact mode of action for Deltamethrin is currently not known. It is generally assumed that pyrethroids affect neuroactivity by delaying the closing of sodium channels resulting in alteration of nerve activity [3,4]. However, in recent years, many effects by Deltamethrin are reported in several epidemiological and experimental studies. Clinical manifestations of acute Deltamethrin poisoning have been documented in occupationally and accidentally exposed cases. Moreover, a number of studies on the side effects of this insecticide have been detailed, including neurotoxicity, immune-suppression and allergy [ 5,6,7], hypertension[8], decreased testosterone levels [9] and genotoxic, immunotoxic and tumorogenic effects of Deltamethrin in mammalian and non-mammalian species [10, 11]. The main reaction involved in the metabolism of Deltamethrin in mice and rats is ester cleavage mainly due to the action of carboxyesterase. Metabolism in fish is largely oxidative and deficient in esterases metabolization [1, 2]. Study of Deltamethrininduced oxidative stress and its influence on various antioxidants could provide useful information on the ecotoxicological consequences of Deltamethrin use [13, 14].

Regarding the possible harmfulness of Deltamethrin and the few studies on this subject, the aims of this work are to evaluate the persistence of Deltamethrin in oil plants treated with the doses of principle active substance recommended by the producers, and to compare the physicochemical characteristics of olive oils extracted from Deltamethrin-treated and untreated plants at different concentrations during an experimental period of 2 months. Thus, this research could review the real risk in the use of this product for plant protection in olive growing. 


\begin{abstract}
II. Materials and methods
2.1. Site study and experimental design

The experiment was conducted in 2012 in the southern Tunisia planted in the experimental fields of OIS - Olive Institut of Sfax (Sfax, Tunisia) subjected to the agronomic management.

Uniform plants with similar degree of infestation, age and fruit yield, were divided in two groups: 50 Deltamethrin-treated plants and 50 untreated plants (control). The active ingredient of Decis (Serbios, Rovigo, Italy), with a weight composition of $10 \%$ was used with a dosage of $0.1\left(C_{1}\right) ; 0.5\left(C_{2}\right) ; 1\left(C_{3}\right) ; 5\left(C_{4}\right) ; 10\left(C_{5}\right) \mu \mathrm{L}$ $\mathrm{L}^{-1}$. The concentration $\mathrm{C}_{3}$ presented the recommended dose of Deltamethrin in olive growing. The product was distributed in a single treatment carried out on 15 September 2012 using a manual spraying machine, giving about $2 \mathrm{~L}$ per plant. Subsequently, random samplings of drupes $(7 \mathrm{Kg})$ were carried out every week for two months after Deltamethrin application. For each date of sampling, an aliquot of $500 \mathrm{~g}$ of drupes was used for the analysis of Deltamethrin residues, while the remaining aliquot of $6.5 \mathrm{Kg}$ was milled in the same day (OIS, Tunisia). The oil so extracted was kept in dark-glass bottles at a temperature of $4{ }^{\circ} \mathrm{C}$.
\end{abstract}

\title{
2.2. Extraction and quantitative analysis of Deltamethrin
}

The sample processing and analysis is described in my previously validated method [15].

\subsection{Physicochemical and nutritional parameters of oil}

2.3.1. Free acidity, peroxide number and specific absorbance at 232 and $270 \mathrm{~nm}\left(\mathrm{~K}_{232}\right.$ and $\left.\mathrm{K}_{270}\right)$

The acidity, expressed as a percentage of oleic acid, the peroxide number (amount of oxygen able to oxidize fatty acid), and the specific absorbance were determined according to the official methods described in European Regulation EC 2568/91 and EC 1989/03 [16,17].

\subsubsection{Fatty acids}

For the determination of fatty acids, $0.15 \mathrm{~g}$ of oil were added to $100 \mu \mathrm{L}$ of a $0.2 \mathrm{~N} \mathrm{KOH} /$ methanol solution. The solution was mixed for $10 \mathrm{~min}$ and the recovered supernatant was suspended in $250 \mu \mathrm{L}$ of hexane and then analyzed by a gas-chromatograph analyses were carried out with Hewlett-Packard 6890 Series GC, Agilent Technology equipped with a Hewlett-Packard 5973 Mass spectrometer, Agilent Technology. The separation of organic compounds was obtained by using a HP- 5MS fused silica capillary column $(\mathrm{L}=60 \mathrm{~m}, \varnothing=$ $0.25 \mathrm{~mm}, 0.25 \mu \mathrm{m}$ film thickness, Agilent Technology). The oven temperature was programmed as follows: $100^{\circ}$ $\mathrm{C}$ hold for $2 \mathrm{~min}$, raised at $5{ }^{\circ} \mathrm{C} / \mathrm{min}$ to $290{ }^{\circ} \mathrm{C}$ (held for $20 \mathrm{~min}$ ). Helium was used as a carrier gas at a flow rate of $1.07 \mathrm{~mL} \mathrm{~min}{ }^{-1}$. The injection was set on a splitless mode at $250{ }^{\circ} \mathrm{C}$. The injected volume was $1.0 \mu \mathrm{L}$, the solvent delay was $6.00 \mathrm{~min}$ and the total run time was $60 \mathrm{~min}$. Detection was conducted by a mass selective detector with electron impact ionization at $70 \mathrm{eV}$ with $2.9 \mathrm{~s}$ scan time over a 50-550 a.m.u. range resolution. Mass spectra were compared to the reference compounds in Wiley $275 \mathrm{~L}$ mass spectral library.

\subsubsection{Total phenol measurement}

Total phenol content of extracts was determined using the Folin-Ciocalteu technique [18]. Briefly, an aliquot of $50 \mu \mathrm{L}$ of different extracts was assayed with $250 \mu \mathrm{L}$ of Folin reagent and $500 \mu \mathrm{L}$ sodium carbonate $(20 \%, \mathrm{w} / \mathrm{v})$. The mixture was vortexes and diluted with water to a final volume of $5 \mathrm{~mL}$. After incubation for 30 $\mathrm{min}$ at room temperature, the absorbance was read at $765 \mathrm{~nm}$ and total phenols in the extracts were expressed as gallic acid equivalents (GAE), using a calibration curve of a freshly prepared gallic acid solution. Eight determinations were performed $(n=8)$. For the gallic acid, the curve absorbance versus concentration is described by the following equation:

$$
y=0.0012 x-0.0345\left(R^{2}=0.999\right)
$$

\subsubsection{Sensory evaluation}

Sensory evaluation was carried out according to the official methods of the IOOC (1996) [19]. Such evaluation monitored the potential presence of any defects that might declass the oil from the best quality designation to a lower one.

\subsection{Statistical analysis}

All experiments were performed in triplicates and mean values are presented. Calculation of average values and analysis of standards deviation (represented as error bars) were performed using Origin lab 6.1. For each essay a control test was performed. Multivariate analyses (Element Coefficient Correlations) and Spearman Test were used to determine the effect of the different concentrations on the physicochemical and nutritional parameters of the olive oil. These studies were performed using the XLSTAT-Pro7.5. A probability of 0.05 or less was considered as statistically significant (95\% confidence interval). 


\section{Results and discussion}

\subsection{Deltamethrin residue monitoring}

Deltamethrin residue was monitored during the whole experiment in order to study its persistence. For more convenience, only $\mathrm{C}_{3}$ (the recommended dose) and $\mathrm{C}_{5}$ (high dose) were analyzed weekly. Results showed that the persistence of Deltamethrin is more important when the recommended dose is exceeded. In fact, the amount of this pyrethroid in the $\mathrm{C}_{3}$ samples decreased of about $50 \%$ two weeks after the treatment and below the MRL after 28 days ( 1.5 and $0.09 \mathrm{mg} \mathrm{Kg}^{-1}$, respectively). For the $\mathrm{C}_{5}$ samples, the residue amount remains much important at the same dates $\left(2.1\right.$ and $\left.1.7 \mathrm{mg} \mathrm{Kg}^{-1}\right)$

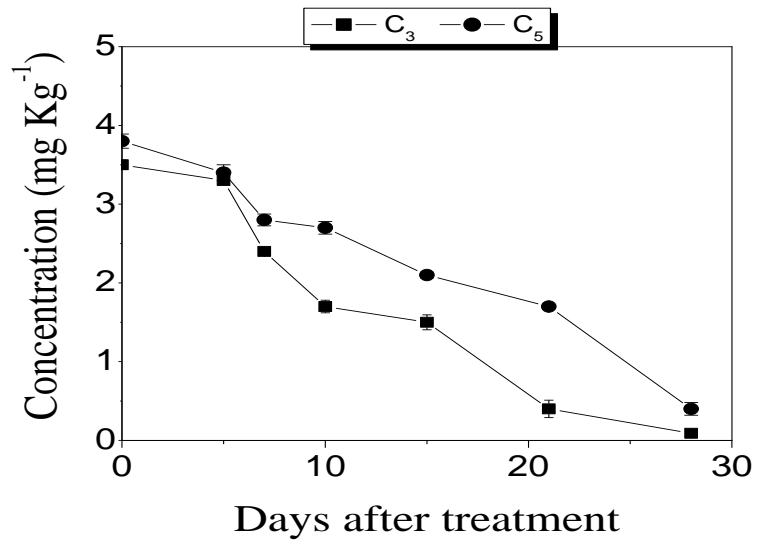

Fig. 1. Concentration of Deltamethrin residue in olive oil obtained from samples treated with $\mathrm{C}_{3}$ (square symbol), and samples treated with $\mathrm{C}_{5}$ (circle symbol) during the experimental period.

The values represent the average $( \pm \mathrm{SD})$ of three measurements for each sample of olive oil.

\subsection{Physicochemical parameters of oil}

During the experimental period the acidity and the peroxide number increased with parallel trends showing significant difference for the highest concentrations $\left(\mathrm{C}_{4}\right.$ and $\left.\mathrm{C}_{5}\right)$ (Fig.2 A and B). At the end of the experimental period the peroxide number reached almost $7 \mathrm{meq} \mathrm{Kg}^{-1}$. The same trend was observed for acidity. In fact, four weeks after the treatment, the difference was important and increased of about $0.5 \%$ of oleic acid for the $\mathrm{C}_{4}$ and $\mathrm{C}_{5}$ (Fig. $2 \mathrm{~A}$ ). The level of polyphenols had, in most cases, parallel trends and declined from 260 to 200 (Fig. 2C).
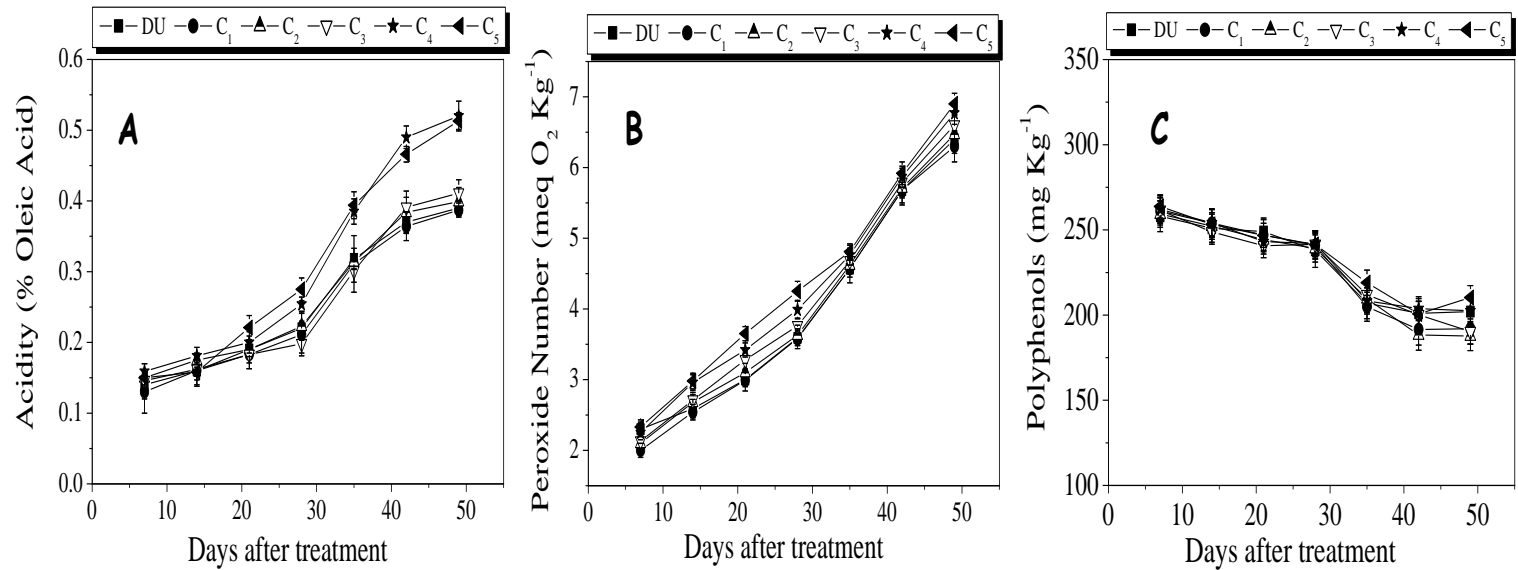

Fig 2. Evolution of the Acidity (A), Peroxide Number (B) and Polyphenols (C) during the experimental period.

The values present the mean of three measurements

$( \pm$ SD) of the olive oil samples $(\mathrm{p} \leq 0.05)$

Although the results show significant difference for the $\mathrm{C} 4$ and $\mathrm{C} 5$, the oil obtained from these samples can be classified as extra-virgin since the values of acidity, peroxide number remain within the standards of the IOOC [19] (Table 1) 
Table 1: Extra virgin olive oil quality standards (according to the CODEX STAN 33-1981 [20])

\begin{tabular}{lc}
\hline Parameter & Standards \\
\hline Free acidity (\%) & $<0.8$ \\
Peroxide number $\left(\mathbf{m e q ~}^{-1} \mathbf{~ g}^{-1}\right)$ & $<20$ \\
Fatty acids $\left(\mathbf{m g ~ K g}^{-1}\right):$ & \\
Mysteric acid & $0-0.5$ \\
Stearic acid & $0.5-5$ \\
Palmitic acid & $7.5-20$ \\
Oleic acid & $55-83$ \\
Linoleic acid & $3.5-20$ \\
Linolenic acid & $<1$ \\
\hline
\end{tabular}

Regarding $\mathrm{K}_{232}$ and $\mathrm{K}_{270}$ parameters, no significant difference is observed between the DeltamethrinUntreated samples and treated ones with the concentrations $\mathrm{C}_{1}, \mathrm{C}_{2}, \mathrm{C}_{3}$. However, the values for $\mathrm{C}_{4}$ and $\mathrm{C}_{5}$ increase to attain 2.68 and 0.35 for the two absorbances, respectively (Table1). Consequently, the standards of the specific absorbances ( 2.5 for $\mathrm{K}_{232}$ and 0.2 for $\mathrm{K}_{270}$ ) are exceeded. This type of analysis can detect the presence of the beginning of oxidative processes that produce a shift double bonds causing formation of the corresponding dienic and trienic conjugated bonds [19].

Table2: $\mathrm{K}_{232}$ and $\mathrm{K}_{270}$ values of the olive oil samples at the end of the experiment

\begin{tabular}{lll}
\hline & $\mathbf{K}_{\mathbf{2 3 2}}$ & $\mathbf{K}_{\mathbf{2 7 0}}$ \\
\hline $\mathbf{D U}$ & 2.45 & 0.25 \\
$\mathbf{C}_{\mathbf{1}}$ & 2.47 & 0.23 \\
$\mathbf{C}_{\mathbf{2}}$ & 2.47 & 0.21 \\
$\mathbf{C}_{\mathbf{3}}$ & 2.49 & 0.27 \\
$\mathbf{C}_{\mathbf{4}}$ & 2.67 & 0.31 \\
$\mathbf{C}_{\mathbf{5}}$ & 2.68 & 0.35 \\
\hline
\end{tabular}

Concerning the fatty acids, the levels of oleic, linoleic, linolenic, palmitic and stearic acids did not show significant differences between Deltamethrin-treated and untreated plants (Fig. 3) and their content remained within the limits normally expected for 'extra-virgin' olive oils [17]. The only significant differences in fatty acids contents between treated and untreated plants regarded the levels of mysteric and oleic acids in the sampling of the two highest concentrations (Fig. 3). In fact, after 30 days from treatment, Deltamethrin-treated plants $\left(\mathrm{C}_{4}\right.$ and $\left.\mathrm{C}_{5}\right)$ had significantly higher levels of mysteric acid if compared to untreated plants $(0.015$ and $0.027 \mathrm{mg} \mathrm{kg}^{-1}$, respectively), oleic acid (3.67 and $3.11 \mathrm{mg} \mathrm{kg}^{-1}$, respectively) and linoleic acid (10.04 and 7.99 $\mathrm{mg} \mathrm{kg}^{-1}$, respectively) (Figs. 3). Despite this difference, the values of these parameters remain within the limits established by the IOOC [19] 

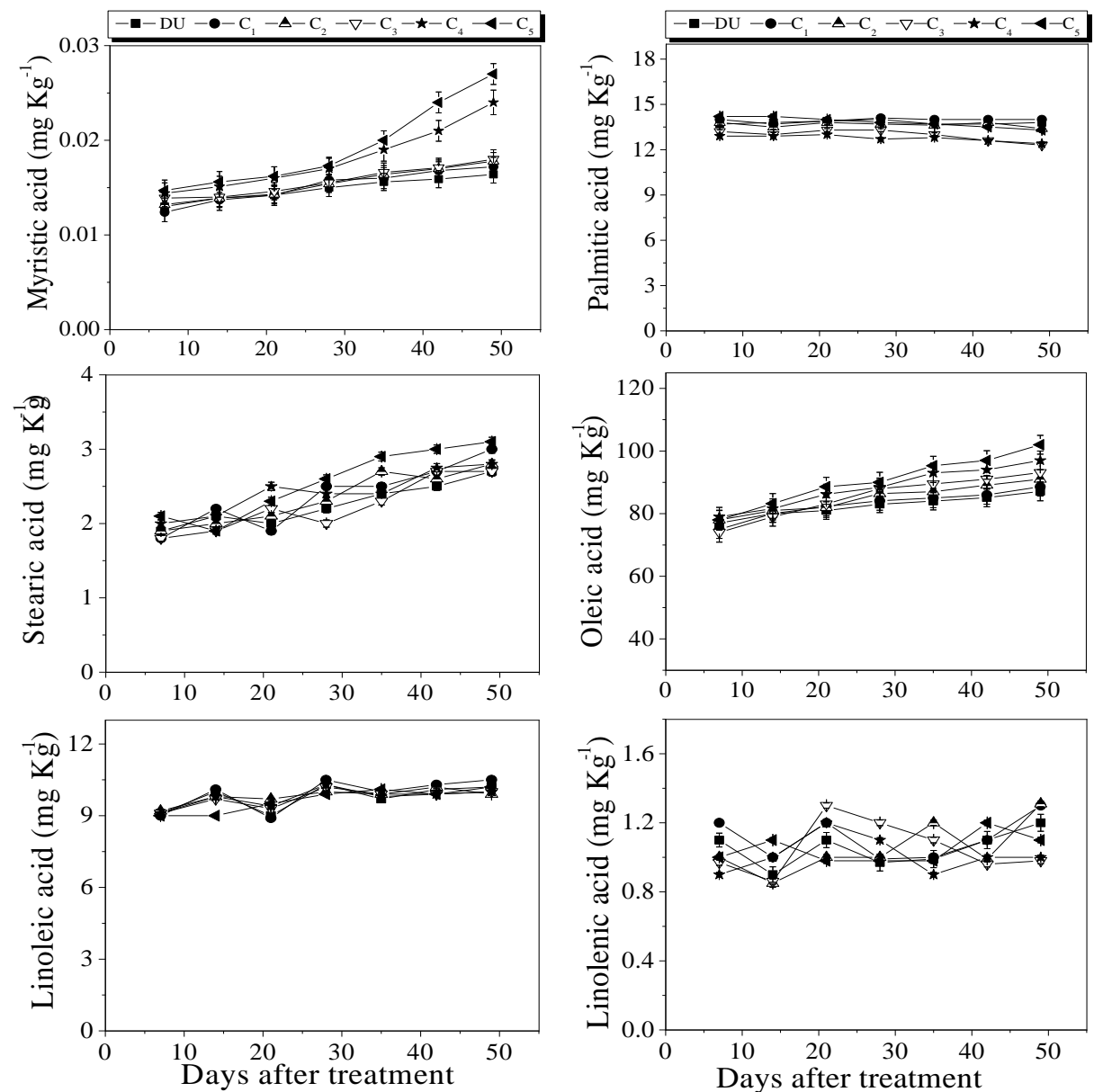

Fig 3. Concentrations of fatty acids during the experimental period, olive oil from Deltamethrin-Untreated samples (DU); olive oil obtained from samples treated with different concentrations.

The values represent the average of three measurements $( \pm S D)$ for each sample $(p \leq 0.05)$.

At the end of the experimental period the sensory evaluation did not reveal significant differences in unpleasant aromatic notes, such as musty, rancid, fusty and muddy flavors, nor defects between Deltamethrintreated with the concentrations $C_{1}, C_{2}$ and $C_{3}$ and untreated plants. In this case, the oil can be defined as "extravirgin" according to EC Regulation 1989/03, with the exception for the oils obtained from samples treated with $\mathrm{C}_{4}$ and $\mathrm{C}_{5}$. The "fruity" aroma was more perceptible for untreated samples, and the "fluid" aspect was more intense for the samples obtained from samples treated with the two highest concentrations.

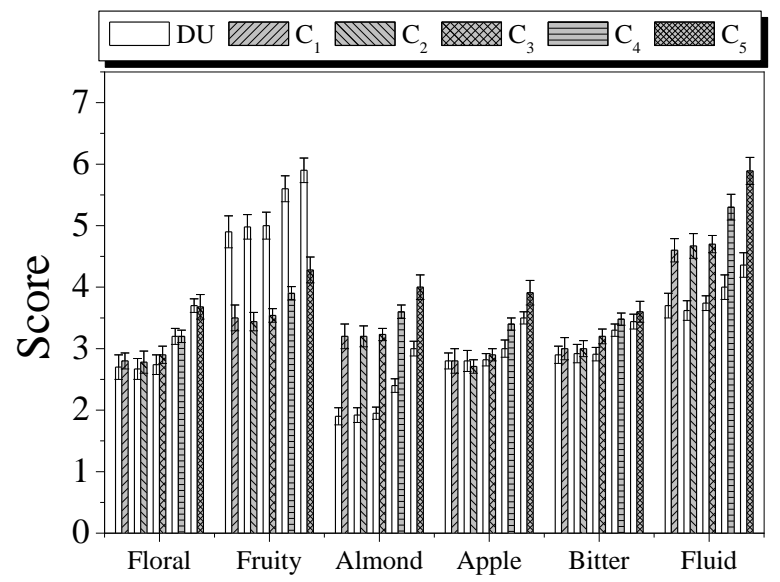

Fig.4. Sensory scores of the olive oil obtained from Deltamethrin-Untreated plants (DU) and Deltamethrin treated plants with the different concentrations $\left(\mathrm{C}_{1}, \mathrm{C}_{2}, \mathrm{C}_{3}, \mathrm{C}_{4}, \mathrm{C}_{5}\right)$. 
According to these results and our previous work [15], we assume that physicochemical characteristics, climatic conditions (essentially sun radiation, rainfall and wind) and dose of treatment affected the kinetic of degradation of Deltamethrin. In case of treatment with recommended dose, we noticed a fast decomposition in the first week and consecutively an important decrease of Deltamethrin residue (50\%) in the first 21 days and remain below the MLRs $\left(0.1 \mathrm{mg} \mathrm{kg}^{-1}\right.$ according to the Codex Alimentarius) until the $28^{\text {th }}$ day. This result is not in agreement with deltamethrin producers who pretend that 7 days after the application are sufficient to harvest the fruits. The more the recommended dose is exceeded the more the active substance persists in the oil (Fig. 1).

The current study confirmed that treatments with Deltamethrin do not affect the physicochemical and nutritional parameters of oils and that the few differences observed in oil physicochemical parameters (Figs. 1,2 and 3) are rather due to the increase of the dose of treatment, meaning that Deltamethrin has no effect if the recommended dose is respected and the results of the sensory evaluation (Fig. 4) demonstrate that both oils from Deltamethrin-treated and untreated plants can be defined as 'extra-virgin' having a good equilibrium of the positive attributes.

However, the recommended dose is often not respected in developing countries. In fact, the unawareness of the farmers and accidents of use of pesticides leaded to an excessive amount of active substance deposited on the plant. In this case, the quality of the oil will be affected since the deltamethrin is a lypophilic substance.

In conclusion, serious doubts remain about the safety and healthiness of oil obtained from fruits treated with Deltamethrin. Consequently, the supposition of the safety of Deltamethrin as it is a biodegradable substance having a quick photolysis should be revised.

\section{References}

[1] A. M. Goomez-Caravaca, L. Cerretani , A. Bendini , A. Segura-Carretero, A. Fernández-Gutiérrez ,M. Del Carlo, D. Compagnone , A. Cichelli , Journal of Agriculture and Food, 56 (12), 2008, 4577-4583

IPCS, Environmental Health Criteria 97; Deltamethrin; Geneva, World Health Organization, 1990, 133 pp

[3] J. R. Corbett, K Wright, A. C. Baillie, The biochemical mode of action of pesticides ( $2^{\text {nd }}$ ed., Academic press, London, 1984)

[4] M. Haruhiko , T. Narahashi, Interaction of Tetramethrin and Deltamethrin at the Single Sodium Channel in Rat Hippocampal Neurons.

[5] H. Hoellinger, A. Lecorsier, D.C. Thang,. The micronucleus test for possible cytogenotoxicity of some pyrethroids. Mutation Research/Environmental Mutagenesis and Related Subjects, 130(3), 1984, 244.

[6] S.H. Kowalczyk-Bronisz, J. Gieldanowski, B. Bubak, Immunological profile of animals exposed to pesticide deltamethrin. Arch. Immunol. Ther. Exp. (Warsz), 38, 1990, 229-238.

[7] J. Lukowicz-Ratajczak, J. Krechniak, Effects of deltamethrin on the immune system in mice. Environmental Research, 59(2), 1992, 467-475.

[8] P.J. Forshaw, J.E. Bradburry,. Pharmacological effects of pyrethroids on the cardiovascular system of the rat. European Journal of Pharmacology, 91(2-3), 1983, 207-213.

[9] M.H. Salem, Z. Abo-Elezz, G.A. Abd-Allah, G.A. Hassan, N. Shaker, Effects of organophosphorus (dimethoate) and prethroid (deltamethrin) pesticides on semen characteristics in rabbits, Journal of Environmental Science and Health B, 1988, 23 (3), 279-290.

[10] R. Hussain, M. Malaviya, P.K. Seth, R. Husain, Effect of deltamethrin on regional brain polyamines and behaviour in young rats. Pharmacology and Toxicology, 1994, 74, 211--215.

[11] S.P. Bhunya, PC. Pati, Effect of deltamethrin, a synthetic pyrethroid, on the induction of chromosome aberrations, micronuclei and sperm abnormalities in mice. Mutagenesis, 5, 1990, 229--332.

[12] J.P. Demoute, A brief review of the environmental fate and metabolism of pyrethroids. Pesticides Sciences, 27, 1989, 375-385. NeuroToxicology, 22(3), 2001, 329-339

[13] S. Khosrow Tayebatia, M.A. Di Tullioa, A. Alberto Riccib, F. Amentaa ,. Influence of dermal exposure to the pyrethroid insecticide deltamethrin on rat brain microanatomy and cholinergic/dopaminergic neurochemistry, Brain Research, 1301, 2009, 80 -188. Huynh T. TFrederic Silvestrea,Bertrand De Meuldera,Jean-Pierre Thomec, Nguyen Thanh Phuongb, Patrick Kestemonta

[14] Combined effects of deltamethrin, temperature and salinity on oxidative stress biomarkers and cetylcholinesterase activity in the black tiger shrimp (Penaeus monodon)

[15] I. Jaabiri, D. Belhaj, N. Turki, M. Kallel, H. Ayadi, M. Ksantini, J. Bouzid, R. Gargouri, Development and method validation for determination of Deltamethrin residue in olive oil using a reversed-phase high performance liquid chromatography, IOSR Journal of Applied Chemistry , 6, 2013, PP 01-08

[16] European Community regulation (EC) No. 2568/91, The Characteristics of Olive Oil and Olive-residue Oil and the Relevant Methods of Analysis, official journal of European Communities, 1991.

[17] European Community regulation (EC) No 1989/03, official journal of European Communities: L295 (13/11/2003)

[18] V.L. Singleton, J.A. Rossi, Colorimetry of total phenolics with phosphomolibdic-phosphotungstic acid reagents, American Journal of Enology and Viticulture, 16, 1965, 144-158

[19] International Olive Oil Council (IOOC), General methodology for organoleptic Assessment of virgin olive oil, IOOC Standard procedure- COI/T.20/Doc. 1996, No13.

[20] Codex STAN 33-1981, norme CODEX pour les huiles d'olive et les huiles de grignons d'olive, 2013 